\title{
Sex-specific crossover patterns in Zebrafish (Danio rerio)
}

\author{
N Kochakpour and PB Moens \\ Department of Biology, York University, Toronto, Ontario, Canada
}

\begin{abstract}
Some species display intersex variation in their rate of meiotic recombination, where recombination is usually suppressed in the heterogametic sex. Although no heteromorphic sex chromosomes have been detected in zebrafish (Danio rerio), genetic analysis has indicated a lower frequency of recombination in males relative to females. Our study of the meiotic recombination pattern in female zebrafish indicates that adult females have only a few meiotic oocytes that are found in groups in the ventral zone of the ovarian surface. We used antibody staining of human mutL homolog 1 (MLH1) protein to mark the sites of putative chiasmata to seek a physical basis for the pattern of recombination and its relative frequency in both sexes. We report that MLH1 foci are found mostly in distal regions of the synaptonemal complexes (SCs) in males, but tend to be more evenly distributed in females.
\end{abstract}

Our cytological analysis yields a ratio of MLH1 foci per chromosome in males versus females of $1: 1.55$. This lower level of recombination in males is in general agreement with previously published results from linkage map analysis. However, the similar ratio of MLH1 foci per unit length of SCs in both sexes demonstrates a correlation between SC length and the frequency of recombination rather than a mechanism that suppresses recombination in males. Thus, chiasma interference seems to provide similar expression in males and females in agreement with the situation in humans, where oocytes with longer SCs display a higher level of recombination that is not a consequence of more closely spaced crossovers along the SCs.

Heredity (2008) 100, 489-495; doi:10.1038/sj.hdy.6801091; published online 5 March 2008

Keywords: zebrafish; meiosis; recombination; chiasmata; MLH1; synaptonemal complex

\section{Introduction}

Prophase of meiosis I can be divided into four morphologically distinguishable substages. In leptotene, proteinacious cores (lateral elements) are formed along the sister chromatids. During zygotene, synapsis is initiated between cores of homologous chromosomes, and central elements of synaptonemal complexes (SCs) begin to form. By mid-pachytene, synapsis and SC formation are complete. Through diplotene, homologs dissociate until the sites of reciprocal recombination remain the only points of attachment. These sites, cytologically detectable as chiasmata at metaphase, can be visualized earlier in mid-pachytene by immunocytology of proteins such as MLH1 (human mutL homolog 1) and MLH3 that are required for maturation of recombination nodules (Jones, 1987, Anderson et al., 1999; Marcon and Moens, 2003). In zebrafish, MLH1 mutants fail to form chiasmata and display univalents in metaphase I resulting in arrest of spermatogenesis and subsequent infertility. Females produce aneuploid gametes, an indication of chromosomal missegregation (Feitsma et al., 2007).

During meiotic recombination, genetic exchange occurs between non-sister chromatids of homologous chromosomes. In some species, the rate of meiotic

Correspondence: N Kochakpour, Department of Biology, York University, 4700 Keele Street, Toronto, Ontario, Canada M3I 1P3.

E-mail: nazfarin@yorku.ca

Received 17 August 2007; revised 12 November 2007; accepted 7 December 2007; published online 5 March 2008 recombination varies between the two sexes, where usually the heterogametic sex has the lower level of recombination (Morgan, 1914). This rule applies to many mammalian species. For example, the average rate of human female to male recombination is $1.6: 1$ over the whole genome (Dib et al., 1996). In many species of fish, despite the absence of a heteromorphic sex chromosome, a significant suppression of recombination is found in males relative to females. In rainbow trout, for instance, the female to male recombination ratio is $3.25: 1.0$ (Danzmann et al., 2005).

To study the relative rate of recombination in zebrafish (Danio rerio), Singer et al. (2002) constructed a genetic map based on male meiosis from androgenetic haploid embryos. This was compared to the female genetic map produced from gynogenetic haploids and to the sex average map using embryos from a natural mating (Postlethwait et al., 1998; Shidoma et al., 1999). Males had a significantly shorter map relative to females and a corresponding dramatically lower frequency of recombination. The genetic map encompassing both sexes was intermediate in length between the sex-specific maps. Moreover, the differences were not equally distributed along the entire map: relative to the female map, most of the male linkage groups were confined to the middle regions where the centromeres are located, suggesting a pericentromeric depression of recombination in male zebrafish (Singer et al., 2002).

The use of immunofluorescence microscopy to visualize antibodies against components of the SC and mouse anti-MLH1 on whole-mount preparations of zebrafish spermatocytes provided physical evidence of 
recombination in zebrafish and elucidated its frequency and pattern. The method has the advantage that it can detect the sites of chiasmata without viability constraints of the gametes (Moens, 2006).

\section{Materials and methods}

\section{Slide preparation}

Ovaries were dissected from young adult females (90-120 days old and $19-22 \mathrm{~mm}$ from head to caudal fin). The ovaries from five females were put into $300 \mu \mathrm{l}$ phosphate-buffered saline and gently passed through a 21-gauge and then a 23-gauge needle with a 3-ml syringe to separate the oocytes. The use of a syringe and needle has the advantage over a pipette in that air pressure in the syringe prevents the cells from sticking, minimizing cell loss. The cells were spun down at $2000 \mathrm{rpm}$ for $3 \mathrm{~min}$ and resuspended in $200 \mu \mathrm{l}$ hypotonic solution (1:2 phosphate-buffered saline: Nanopure water) and placed on a slide. After settling for $20 \mathrm{~min}, 200 \mu \mathrm{l}$ of $4 \%$ paraformaldehyde in hypotonic solution with $0.06 \%$ sodium dodecyl sulfate was added to the slide. The liquid was reduced by partial evaporation at room temperature in open air for about $5 \mathrm{~h}$ or about $2 \mathrm{~h}$ under vacuum. (Complete evaporation resulted in a strong background.) The slides were then washed three times for $1 \mathrm{~min}$ each in $0.4 \%$ Photo-Flo 200 (Kodak, Rochester, NY, USA).

Ovaries of 10 adult females (6 months or older) were dissected and incubated with agitation in $10 \mathrm{ml}$ of $0.2 \%$ collagenase in $1.3 \%$ Dulbecco's Modified Eagle's Medium (Sigma, Oakville, Ontario, Canada) for $20 \mathrm{~min}$ at $32^{\circ} \mathrm{C}$. The cells were then washed with Dulbecco's Modified Eagle's Medium and incubated in 0.05\% trypsin + Dulbecco's Modified Eagle's Medium for $10 \mathrm{~min}$ at $32{ }^{\circ} \mathrm{C}$ on the shaker. The cells were kept on the shaker for another $10 \mathrm{~min}$ with $1 \%$ trypsin inhibitor and bovine serum albumin to inactivate the trypsin. The cell suspension was pipetted up and down with a $10 \mathrm{ml}$ plastic pipette 100 times and passed through a $100-\mu \mathrm{m}$ mesh. The flow-through liquid was spun down at $2000 \mathrm{rpm}$ for $3 \mathrm{~min}$ and resuspended in $200 \mu \mathrm{l}$ hypotonic solution and placed on a slide. After settling for $20 \mathrm{~min}$, $200 \mu \mathrm{l} 4 \%$ paraformaldehyde in hypotonic solution with $0.06 \%$ sodium dodecyl sulfate was added to the slide. The liquid was reduced by partial evaporation at room temperature for about $5 \mathrm{~h}$ in open air or about $2 \mathrm{~h}$ in a vacuum. The slides were then washed three times, $1 \mathrm{~min}$ each in $0.4 \%$ Photo-Flo 200.

\section{Cryosections}

To prepare tissue for cryostat sectioning, dissected ovaries were mounted in tissue-freezing medium (TBS; Electron Microscopy Sciences, Hatfield, PA, USA) on aluminum blocks. Samples were frozen in isopentane immersed in liquid nitrogen for $30 \mathrm{~s}$ and kept on dry ice to prevent subsequent thawing and freezing. Ovaries were cut at $-16{ }^{\circ} \mathrm{C}$ and were picked up on a roomtemperature slide. After collecting the required sections, the slide was immersed in $-20^{\circ} \mathrm{C}$ acetone for $30 \mathrm{~s}$ and in phosphate-buffered saline for $5 \mathrm{~min}$. Sections were then fixed in $2 \%$ paraformaldehyde, washed three times in $4 \%$ Photo-Flo for $1 \mathrm{~min}$ each, air-dried and used for antibody staining.

\section{Immunostaining}

After drying, the cells were blocked in 10\% ADB antibody dilution buffer (10\% goat serum, 3\% bovine serum albumin, and $0.05 \%$. Triton X-100 in phosphatebuffered saline) and incubated in primary antibodies for $3 \mathrm{~h}$ or overnight. After another set of washing and blocking, the cells were incubated with secondary antibodies for $1 \mathrm{~h}$ at $37^{\circ} \mathrm{C}$. The slides were washed and dried and mounted in ProLong Antifade mounting agent (Molecular Probes, Eugene, OR, USA) and covered with a coverslip. Dilutions of 1:120 of rabbit antibodies against the lateral and central elements of hamster SCs (SYCP3 and SYCP1, respectively) were used to visualize SCs. MLH1 protein was detected by 1:35 dilutions of commercial mouse monoclonal antibody (BD Bioscience Pharmingen, Franklin Lake, NJ, USA).

\section{Results}

\section{Meiotic oocyte localization}

Our first attempts to obtain meiotic oocytes from adult females failed. This, in addition to the lack of evidence in the literature for continuing meiosis in adult zebrafish, prompted us to investigate whether meiosis is indeed continuous in adults. We looked at the morphology of whole-mount preparations and serial cryosections of ovaries of young adult and adult females.

The whole-mount preparation of ovaries of a young adult female displays a lobed appearance (Figure 1). This morphology is not as apparent in adult fish, likely due to the presence of many vitellogenic oocytes.

Primary oocytes were located in the ventral side of the ovary with detectable strands of SCs in the nuclei when transversed cryostat sections of intact ovary of an adult fish were used. Secondary and vitellogenic oocytes were detectable at the periphery of the oocytes in follicular nuclei stained with 4,6-diamidino-2-phenylindole. The primary oocytes with meiotic nuclei were found in groups of two to six among oocytes in other stages in the ventral zone of the ovarian surface (Figures 2a-c). Strands of SCs are visible in Figures $2 a^{\prime}-c^{\prime}$, and the corresponding inserts in higher magnification. Usually, only one or two clusters of oocytes were seen in each cross section in a few consecutive serial sections. Subsequent sections had no oocytes and new clusters appeared in later sections along the longitudinal length of ovary in both young and adult zebrafish.

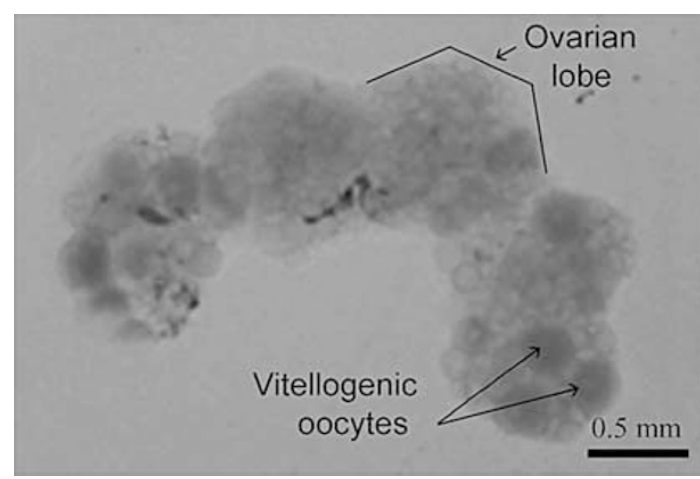

Figure 1 Whole-mount preparation of an ovary dissected from a young adult female zebrafish shows a lobed appearance. 

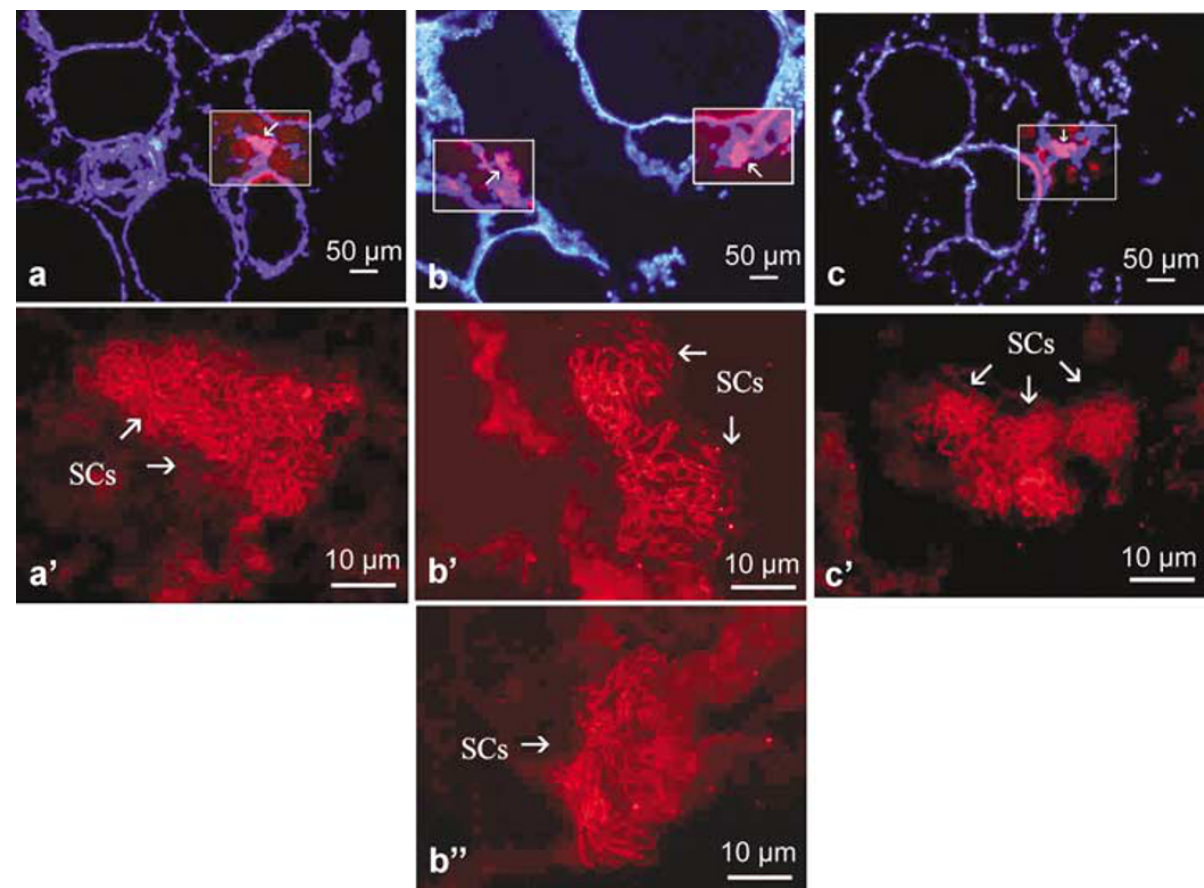

Figure 2 Cryostat cross-sections of the ovary taken with $\times 10$ objective lens (a-c) and at higher magnification in inserts $\mathbf{a}^{\prime}, \mathbf{b}^{\prime}, \mathbf{b}^{\prime \prime}$ and $\mathbf{c}^{\prime}$ Synaptonemal complexes (SCs) are visualized in red using rabbit anti-SYCP1 antibody (central elements of SC). Nuclei of follicular cells surrounding oocytes are stained with 4,6-diamidino-2-phenylindole (blue). Primary oocytes are found in groups of 2-6 among oocytes in other stages. Usually, only one or two batches of oocytes are seen in each transverse section of ovary.

The fragmented pattern of meiotic oocytes observed along the longitudinal length of the ovary of adult fish in addition to the lobed appearance of the ovary in young females suggests that one or two nests of meiotic oocytes exist in each of six lobes along the ovary. This is in agreement with our observations on meiotic spreads where only a few meiotic cells can be obtained from each female.

\section{Meiotic karyotype}

The whole-mount spread preparations of meiotic oocytes showed 25 SCs with no differentiated sex chromosomes in each oocyte. As SC lengths vary in different substages of prophase, only oocytes in midpachytene were used for measurements. Mid-pachytene stage was defined by the presence of at least one MLH1 focus on each SC. Six nuclei collected from 10 young adult females (90-120 days) and 3 nuclei obtained from 10 adult fish (6 months or older) were used to determine the length of the SCs as well as the distribution pattern of recombination.

Generally, only a few oocytes can be collected from individual females, some of which are lost during processing. Sample size was further reduced, because only nuclei in mid-pachytene with clear and measurable SCs and MLH1 foci could be used for analyses. Oocytes of 10 young and 10 adult females were collected to obtain the 9 nuclei that were analyzed in this study.

The lengths of SCs of 9 nuclei measured with Image Pro software indicated consistently longer SCs in female zebrafish verses males. Zebrafish spermatocyte data were used from a previous study in our lab (Moens, 2006). Both studies used the same measuring protocol and similar spread preparation techniques making the data obtained for the two sexes comparable. The total length of SCs of spermatocytes varied from 230 to $260 \mu \mathrm{m}$; the total length of female SCs varied between 280 and $485 \mu \mathrm{m}$, indicating greater variation in the total length of SCs in oocytes compared with spermatocytes. Electron microscopy studies of SCs in male and female zebrafish have also shown longer SCs in females compared with males with more extensive variations among oocytes (Wallace and Wallace, 2003).

\section{Rates and patterns of recombination}

Putative crossovers are associated with MLH1 proteins and are visualized as foci along the SC in whole-mount nuclear spread preparation of oocytes and spermatocytes (Figure 3). In zebrafish spermatocytes (Moens, 2006), most SCs have a single MLH1 focus, but one or two SCs may have a second focus. Therefore, in each spermatocyte nucleus, there are 25-27 MLH1 foci (Figure 3a). However, in females, the total number of MLH1 foci per nucleus varied from 33 to 40 . Figures $3 \mathrm{~b}$ and c show two cells with 33 and 40 MLH1 foci with a total SC length of 282 and $485 \mu \mathrm{m}$, respectively.

Figure 4 is a graphical presentation of the distribution of MLH1 foci along SCs in the two oocytes mentioned above. SCs are illustrated as bars arranged according to their length, and each MLH1 focus is shown as a black line on the bars. Often MLH1 foci are located near the termini of the SCs. This is more obvious in oocytes with fewer MLH1 foci (Figure 4a). However, in oocytes with more MLH1-associated sites, several foci are seen in the medial regions (Figure $4 b$ ).

We studied the pattern of recombination in 154 SCs from 9 nuclei, as described previously. These SCs were equalized in length in that each SC length was divided in 

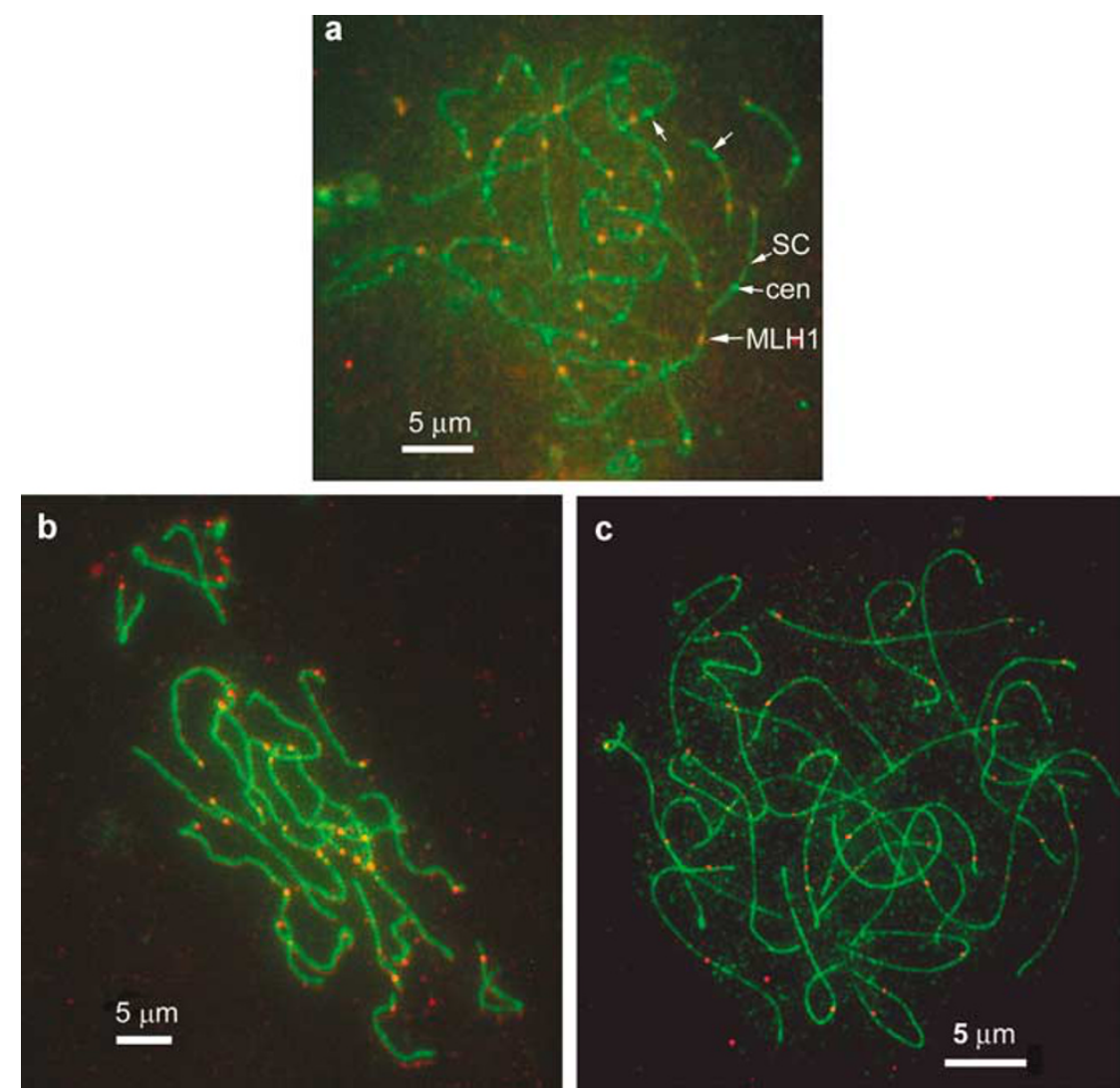

Figure 3 Immunofluorescent microscopy of a spermatocyte nucleus (a) and two oocyte nuclei with 33 (b) and 40 (c) MLH1 foci. Synaptonemal complexes: labeled fluorescent green with FITC; MLH1 foci: labeled fluorescent red with rhodamine. MLH1, human mutL homolog 1 .

half, and each half was again divided into 10 equal segments of $5 \%$ of the total SC length. The number of MLH1 foci found in each 5\% segment was counted and is shown in Figure 5a. The resultant diagram was compared to that of the male adapted from Moens (2006) (Figure 5b). Centromeres of both male and female zebrafish are not consistently detectable, perhaps due to the state of chromatin decondensation as suggested by Wallace and Wallace (2003). Owing to the abundance of spermatocytes collected from a pair of testes, a reasonable number of nuclei with detectable centromeres can be obtained. In the study by Moens (2006), nuclei with detectable centromeres were selected, and SCs were equalized based on their short and long arms. As we were unable to collect sufficient nuclei in females that displayed centromeres, it was necessary to alter the male data, so that a direct comparison to the female data was possible. Generally, SCs tended to be longer in the nuclei in which centromeres were not visible. Wallace and Wallace (2003) also reported that centromeres are either visible on all the SCs of a pachytene nucleus or none, which they interpreted to be a result of developmental sequence of the nuclear growth and/or chromatin decondensation.

Closer inspection reveals that only 15\% (38 out of 250) MLH1 foci are located within the first $5 \%$ of the SCs in oocytes, whereas, in spermatocytes, almost $48 \%$ (70 out of 147) of the crossovers occurred in the first $5 \%$ of the ends. Only $3.4 \%$ of MLH1 foci occur in the middle $30 \%$ of the SC in males, whereas in females the value is $14 \%$, suggesting a more equal distribution of chiasmata along the SCs of females relative to males. This agrees with the pericentromeric depression of recombination reported by Singer et al. (2002) in males where the chromosomes are all submetacentric. Very few MLH1 foci are found in the medial region of SCs in these spermatocytes (Moens, 2006).

To confirm that this general pattern is true for whole cells rather than individual SCs, the same analysis was performed on three complete nuclei with 33,36 and 40 MLH1 foci and total SC length of 282, 306 and $485 \mu \mathrm{m}$, respectively. The resultant graphs suggest that generally the chance of finding crossovers in the medial region of the SCs increases with the total nuclear length of SC (Figures $6 \mathrm{a}-\mathrm{c}$ ). Combining the three graphs gives a similar pattern for crossover distribution as that resulting from the analysis of 154 SCs (Figure 6d). A comparison of the number of MLH1 foci in females (250 MLH1 foci in 154 SCs) to males (147 MLH1 foci in 140 SCs) results in a recombination ratio of $1.55: 1$ - a higher recombination frequency in females in general agreement with genetic mapping data (Singer et al., 2002). However, calculating the number of crossovers per unit length of SCs displays almost the same ratio in both sexes. This ratio is 0.107 in males, calculated by dividing 147 MLH1 foci by the total SC length of $1372 \mu \mathrm{m}$, and in females, is 0.106 calculated by dividing 250 MLH1 foci by the total SC length of $2353.12 \mu \mathrm{m}$. 


\section{Discussion}

Immunofluorescence microscope analysis of cryostat cross-sections of ovaries obtained from either young or adult zebrafish demonstrates few meiotic oocytes in 6-7 sequential serial longitudinal sections in the ventral zone of each ovary, suggesting that one or two nests of meiotic
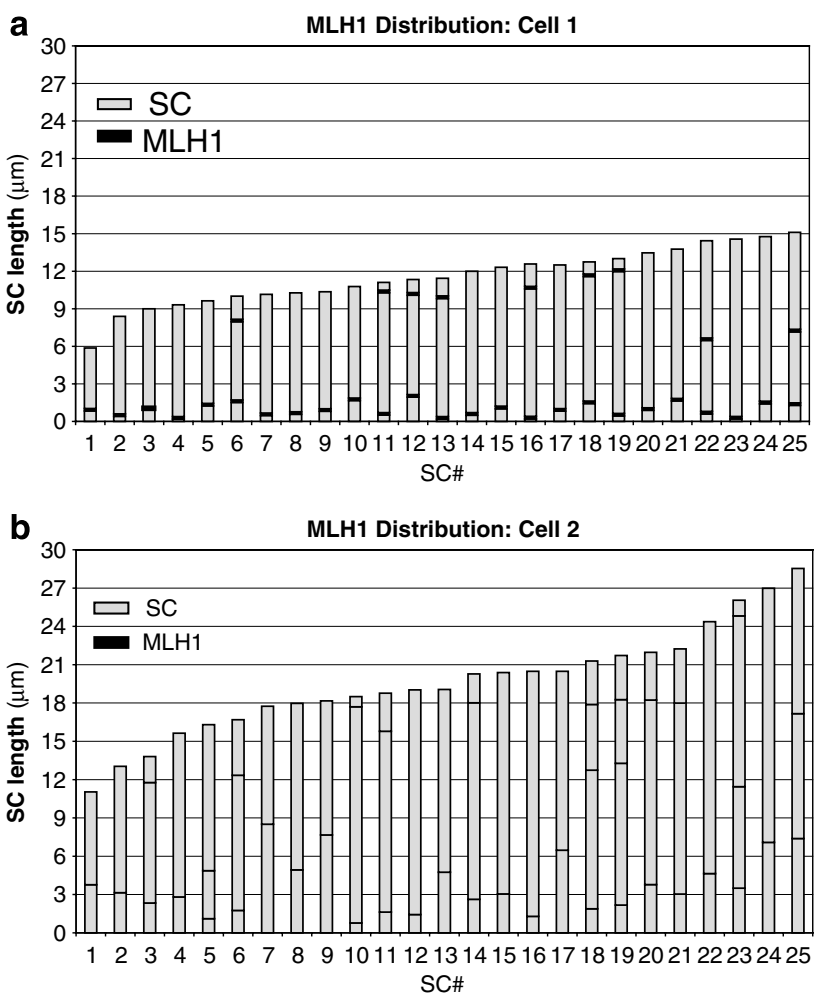

Figure 4 Graphical presentation of the 25 SCs and MLH1 foci of the two oocytes of Figure 3. Cells 1 and 2 represent the oocytes with 33 and 40 MLH1 foci, respectively. Green bars represent SCs and the positions of MLH1 foci are marked in black. In cell 1, nine SCs have two MLH1 foci and in cell 2, eight SCs have double MLH1 foci and three SCs show a third focus. $x$ axis: SC numbers arranged by increasing length; $y$ axis: SC length in $\mu \mathrm{m}$. MLH1, human mutL homolog 1; SC, synaptonemal complex. oocytes exist in each of six lobes along the ovary, as well as confirming continuous occurrence of meiosis in adult females.

Genetic analysis of meiotic recombination indicates a dramatic reduction in the rate of recombination in males relative to females (Singer et al., 2002). Cytological analysis of meiotic recombination reveals a lower frequency of recombination based on fewer MLH1 foci per spermatocyte relative to oocytes. However, the number of MLH1 foci per unit length of SCs indicates the same ratio in both sexes, suggesting that this lower level of recombination in males should not be interpreted as a suppression of recombination in males contrary to the suggestion of Singer and co-workers from genetic linkage analysis. We interpret these data to suggest that the mechanism of recombination in males and females is the same, but more crossovers are found in oocytes compared with spermatocytes because of the greater SC length of females relative to males.

Oocytes with different numbers of crossovers and different lengths of SCs can serve as an intra-sex support for this observation. There is a correlation between numbers of MLH1 foci and SC lengths in nuclei of oocytes, where longer SCs have generally more MLH1 foci distributed more equally along the bivalent. The same conclusion has been drawn from the analysis of human oocytes and spermatocytes, in which oocytes have longer SCs and display higher level of recombination (Tease and Hulten, 2004). In zebrafish, male and female chromosomes having same DNA content display different SC lengths based on consistently longer SCs in females. Thus, the frequency of recombination in each SC correlates with the length of SC rather than the size or DNA content of the chromosome. This is quite interesting, as it suggests that the cytological/physical distance of the SCs reflects the genetic distance, a reasonable observation considering that recombination occurs along the SCs. In another study, frequency of recombination (MLH1 foci) and the length of SCs of two chromosomes with similar sizes were compared in human spermatocytes. Striking difference in the SC length of the two chromosomes was correlated with their different genetic lengths (Lynn et al., 2002). The length of SCs can be determined by the state of DNA packaging of the meiotic
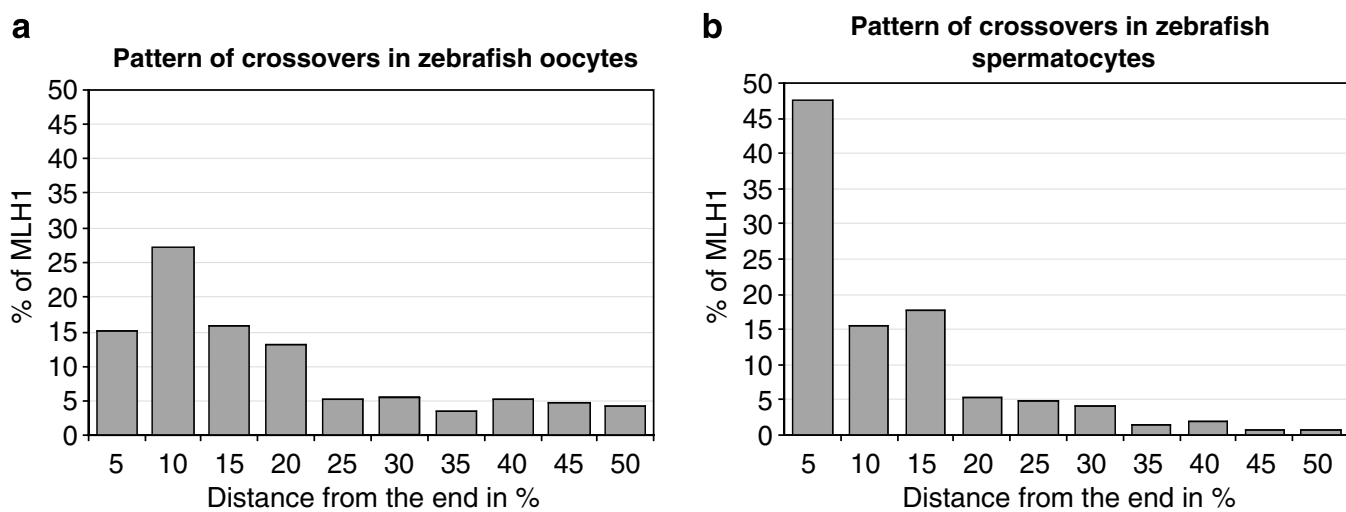

Figure 5 A comparison of the frequency and distribution pattern of crossovers between spermatocytes and oocytes. Each bar represents the number of MLH1 found in 5\% lengths of SC measured from either end to the middle of the SC. On the $x$ axis, ' 0 ' represents either SC end and ' 50 ' represents the middle of the SC. (a) Two hundred and fifty MLH1 foci in 154 female SCs, (b) 147 MLH1 foci in 140 male SCs. MLH1, human mutL homolog 1; SC, synaptonemal complex. 

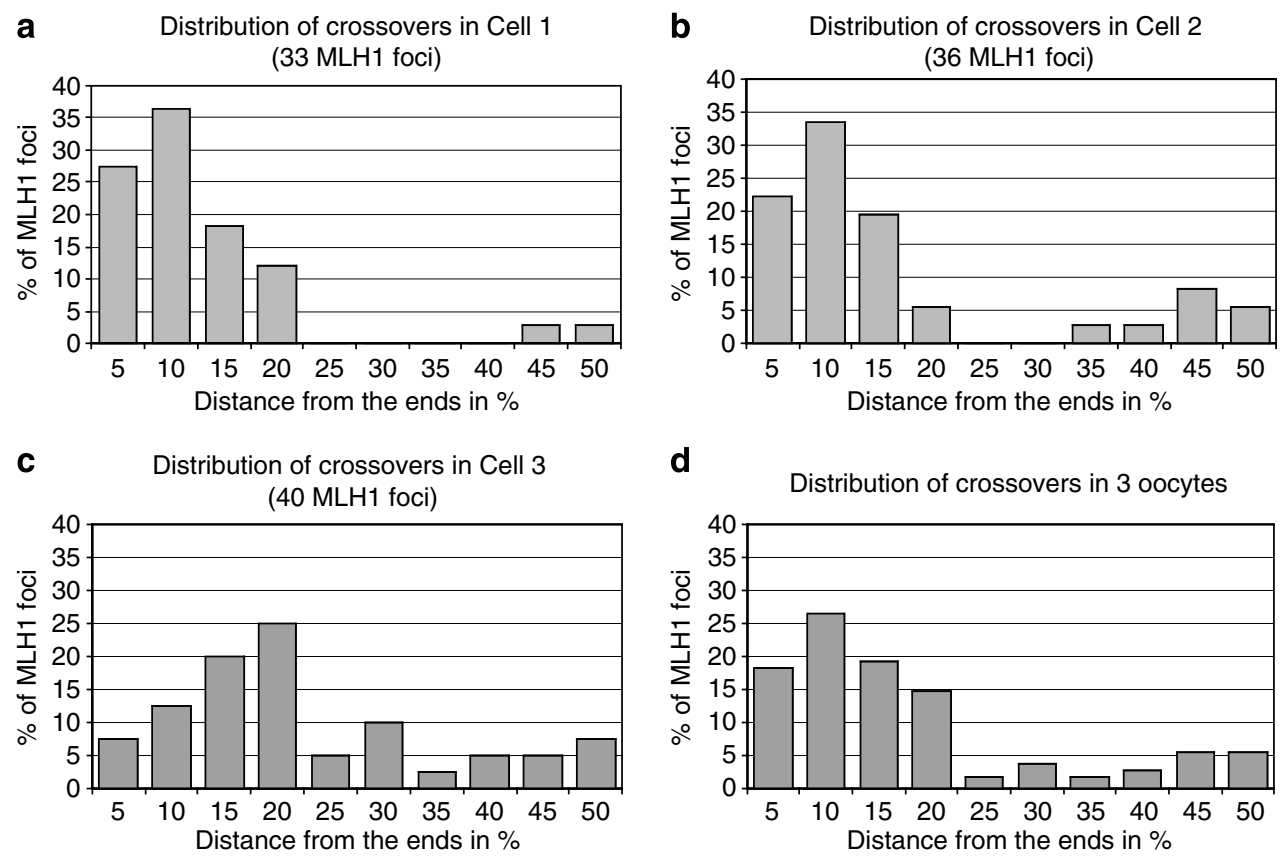

Figure 6 Frequency and distribution pattern of crossovers in three oocytes with 33, 36 and 40 MLH1 foci and total synaptonemal complex length of 282, 306 and $485 \mu \mathrm{m}$, respectively (a-c). (d) Pattern of recombination based on the combined three oocytes. MLH1, human mutL homolog 1 .

chromosomes. The size and compaction of DNA loops could be one possible factor in determining the lengths of the SCs. Considering two chromosomes with the same DNA size, but different DNA packaging, the chromosome with shorter DNA loops and/or less compact loops is expected to be longer and have a longer SC. A preliminary investigation of SC loop size by fluorescence in situ hybridization indicates that, in humans, loops are likely shorter in oocytes than in spermatocytes (Tease and Hulten, 2004).

As concluded from the analysis of nuclei in different species, in early zygotene, SC formation tends to initiate at the ends of the chromosome. Additional sites of synaptic initiation may occur depending on the length of SCs in different organisms. In humans, for example, oocytes display double the number of interstitial initiations relative to spermatocytes, in correlation with their twofold longer SCs (Zickler and Kleckner, 1999). Similarly, more interstitial initiations are expected in zebrafish oocytes with their longer SCs than in spermatocytes, where SCs begin to form at the chromosome ends. Therefore, in males, the end regions of the SCs are the first sites where recombination nodules appear, and the formation of other recombination nodules is inhibited by interference by the MLH1 foci found at the distal region of the SCs in spermatocytes. In oocytes, additional synaptic initiation opportunities in the medial region of the longer bivalents may permit crossovers in the middle of an SC. As SCs are generally longer in oocytes, more synaptic initiation sites are expected in females in agreement with the appearance of more MLH1 foci in the medial region of the SCs. Therefore, we suggest that the pattern of SC formation and interstitial synaptic initiations may correlate with the distribution pattern of crossovers along SCs.
In conclusion, this study provides physical evidence of the pattern of recombination in female zebrafish and confirms a higher recombination frequency in males relative to females. It also suggests that higher level of recombination in females correlates with the longer SCs present in oocytes. We also illustrate that meiotic oocytes are localized in groups in the ventral region of the ovarian surface. This localization of oocytes in the ovary can explain why very few oocytes can be obtained from a single female.

\section{References}

Anderson LK, Reeves A, Webb LM, Ashley T (1999). Distribution of crossing over on mouse synaptonemal complexes using immunofluorescent localization of MLH1 protein. Genetics 151: 1569-1579.

Danzmann RG, Cairney M, Davidson WS, Ferguson MM, Gharbi K, Guyomard R et al. (2005). A comparative analysis of the rainbow trout genome with 2 other species of fish (Arctic charr and Atlantic salmon) within the tetraploid derivative Salmonidae family (subfamily: Salmoninae). Genome 48: 1037-1051.

Dib C, Faure S, Fizames C, Samson S, Drouot N (1996). A comprehensive genetic map of the human genome based on 5264 microsatellites. Nature 380: 152-154.

Feitsma H, Leal MC, Moens PB, Cuppen E, Schulz RW (2007). Mlh1 deficiency in zebrafish results in male sterility and aneuploid as well as triploid progeny in females. Genetics 175: 1561-1569.

Jones GH (1987). Chiasmata. In: Moens PB (ed). Meiosis. Academic Press: Burlington Mass. pp 213-244.

Lynn A, Koehler KE, Judis L, Chan ER, Cherry JP, Schwartz S et al. (2002). Covariation of synaptonemal complex length and mammalian meiotic exchange rates. Science 296: 2222-2225.

Moens P (2006). Zebrafish: chiasmata and interference. Genome 49: 205-208. 
Marcon E, Moens P (2003). MLH1p and MLH3p localize to precociously induced chiasmata of okadaic-acid-treated mouse spermatocytes. Genetics 165: 2283-2287.

Morgan TH (1914). No crossing over the male Drosophila of genes in the second and third pair of chromosomes. Biol Bull 26: 195-204.

Shidoma N, Knapik EW, Ziniti J, Sim C, Yamada E (1999). Zebrafish genetic map with 2000 microsatellite markers. Genomics 58: 219-232.

Singer A, Perlmana H, Yana Y, Walkera C, Corley-Smitha G, Brandhorstb B et al. (2002). Sex-specific recombination rates in zebrafish (Danio rerio). Genetics 160: 649-657.
Postlethwait JH, Yan Y-L, Gates M, Horne S, Amores A (1998). Vertebrate genome evolution and the zebrafish gene map. Nat Genet 18: 345-349.

Tease C, Hulten MA (2004). Inter-sex variation in synaptonemal complex lengths largly determine the different recombination rates in male and female germ cells. Cytogenet Genome Res 107: 208-215.

Wallace BM, Wallace H (2003). Synaptonemal complex karyotype of zebrafish. Heredity 90: 136-140.

Zickler D, Kleckner N (1999). Meiotic chromosomes: integrating structure and function. Annu Rev Genet 33: 603-754. 